\title{
ERK1/2 contributes negative regulation to STAT3 activity in $H S S$-transfected HepG2 cells
}

\author{
Ze Jun TIAN ${ }^{2}$, Wei $\mathrm{AN}^{1, *}$ \\ ${ }^{1}$ Department of Cell Biology, Capital University of Medical Science, 10 Xitoutiao, You An Men Beijing 100054, China. \\ ${ }^{2}$ Department of Cardiology, Second Hospital, Hebei Medical University, Shijiazhuang, China..
}

\begin{abstract}
Signal transducer and activator of transcription 3 (STAT3) is a recently characterized transcription factor which is essential to liver regeneration. We have previously reported that hepatic stimulator substance (HSS), a novel growthpromoting substance, phosphorylated the epidermal growth factor (EGF) receptors and activated downstream RasMAP kinase (extracellular signal-regulated kinases, ERK1/2) cascade. However, whether HSS signal is related to STAT3 pathway remains unclear. The present study is aiming to explore the regulatory effect of activation of ERK $1 / 2$ evoked by HSS on STAT3 phosphorylation and STAT3 signaling. Human hepatoma cell line HepG2 was stably transfected with HSS cDNA and HSS expression was measured by Northern blot. The results showed that the transfection of HSS into HepG2 resulted in remarkable increase in cellular proliferation as compared with the non-transfected cells, and it was further proved that the cellular proliferation in the HSS-transfected cells was related to ERK1/2 activation. Treatment of the cells with $50 \mu \mathrm{M}$ of PD98059, an ERK1/2 specific upstream inhibitor, resulted in ERK1/2 inactivation completely. Inhibition of ERK1/2 allowed the tyrosine of STAT3 to be phosphorylated in a dose-dependent manner to PD98059. Furthermore, transient transfection of STAT3 mutant (STAT3S727A) into HSS-bearing cells could remarkably reverse the inhibitory effect of ERK1/2 on STAT3 phosphorylation. Based upon these results, it is concluded that ERK1/2 negatively modulates STAT3 phosphorylation and this function is dependent on residual serine-727 (S727) of STAT3.
\end{abstract}

Keywords: hepatic stimulator substance, ERK1/2, STAT3, hepatocyte growth.

\section{INTRODUCTION}

The liver is characterized by its extraordinary capacity to regenerate after surgical resection of hepatic lobes or $\mathrm{viral} / \mathrm{chemical}$ injury[1]. The liver regeneration is precisely regulated by a variety of growth factors and cytokines. Despite intense study, the molecular mechanisms dealt with this process remain relatively unclear. However, it has recently been found that at least two distinct intracellular signaling pathways, mitogen-activated protein kinase (MAPK) pathway and Janus kinases-signal transducer and activator of transcription (JAK-STAT) pathway, are involved in the processe of liver regeneration[2]. Extracellular signal-regulated kinases 1/2 (ERK1/2) are major members of MAPK family. In MAPK pathway, extracellular growth molecules bind to their specific receptors on the cell membrane, leading to sequential activation of Ras, Raf, MEK and ERK $1 / 2$. While in JAK-STAT signaling pathway, it has been known that the cytokines (e.g. IL-6) bind to their receptors and activate the cytoplasmic kinases, Janus

${ }^{*}$ Correspondence: Wei AN,

Tel: 0086-10-63291921, Fax: 0086-10-63051130;

E-mail: anwei@cpums.edu.cn kinases (JAKs). The JAKs then phosphorylate and activate a set of latent gene regulatory proteins called signal transducer and activator of transcription (STAT) [3]. STAT3 belong to the family of STAT transcription factors which play a role in cellular responses to a variety of cytokines and growth factors. STAT3 are normally latent in the cytoplasm and activated through phosphorylation on their conservative tyrosine residue Tyr705. The phosphorylated STAT3 are subsequently translocated into nucleus, where they initiate the transcription of some specific genes essential to liver regeneration[4].

Hepatic stimulator substance (HSS) is considered as a novel liver-specific mitogen candidate. In contrast to other non-liver specific growth factors such as epidermal growth factor (EGF) [5], transforming growth factor- $\alpha$ (TGF- $\alpha$ ) [6] and hepatocyte growth factor (HGF)[7], HSS exclusively promotes the cell growth in liver cells or liver-derived tumor cells. We have previously reported that the mitogenic effect of human HSS on hepatocytes growth was related to the up-regulation of EGF receptor phosphorylation on cell membrane and the subsequent activation of intracellular signaling molecules such as Ras and ERK1/2 [8-10]. Recently, we found that in hepatocellular carcinoma cell 
BEL-7402, the addition of recombinant HSS could promote the cellular proliferation with a consistant increase in ERK1/2 activation[11]. In order gain insight into HSS potential action on STAT3 and EKR1/2, we established the HSStransfected HepG2 cells and observed the sustained activation of ERK1/2. The influences of ERK1/2 activity on STAT3 were analyzed by PD98059 inhibition and STAT3S727A dominant negative method. We identified that the transfection of HSS into HepG2 cells increased ERK1/2 activity significantly, but the tyrosine phosphorylation of STAT3 remained unchanged. However, if ERK1/2 activity was pre-inhibited by PD98059, STAT3 tyrosine phosphorylation was greatly enhanced. Additionally, mutation in STAT3 (S727A) resulted in dissociation of ERK1/2 inhibition on STAT3. In this circumstance, STAT3S727A could be directly activated by HSS. These results indicate that the HSS-induced ERK1/2 activation exerts a repressive effect on STAT3 phosphorylation.

\section{MATERIALS AND METHODS}

\section{Materials}

Human hepatoma HepG2 cells were from ATCC (Rockville, MD). TRIzol was from GIBCO/BRL. Random primer labeling kit was from Promega. ERK1/2 antibodies (phospho- Thr202/Tyr204 and non phosphorylated type), STAT3 antibodies (phospho-Tyr705 and non phosphorylated type) and ERK selective inhibitor, PD98059 (2'-amino-3-methoxylfavone), were from Cell Signaling Technology. Horseradish peroxidase-labeled secondary antibody was from Jackson. Western blotting luminol reagent was from Santa Cruz Biotechnology. STAT3 constitutive inhibitor, AG490 [ $\alpha$-cyano-(3, 4-dihydroxy)-N-benzylcinnamide], was from CalBiochem. DNA transfection reagent, N-[1-(2,3-dioleoyloxy)propyl]-N,N,Ntrimethylammonium methylsulfate (DOTAP), was from Roche Diagnostics. Bicinchoninic acid (BCA) protein assay kit was from Pierce. STAT3S727A mutant construct was gifted by Dr. Toshio Hirano, Osaka University, Japan.

\section{Cell culture and DNA transfection}

HepG2 cells were cultured in Dulbecco's Modified Eagles Medium (DMEM) supplemented with $10 \%$ fetal calf serum (FCS) at $37^{\circ} \mathrm{C}$ in a $5 \% \mathrm{CO}_{2}$ and $95 \%$ atmosphere humidified incubator. A total of $1 \times 10^{5} \mathrm{HepG} 2$ cells were allowed to grow to $50-70 \%$ of confluence. For stable transfection[12], the cells were transfected with $5 \mu \mathrm{g}$ of HSS-pCDNA 3.1 or pCDNA 3.1 alone with the mediation of DOTAP following the manufacturer's manual. After transfection for $8 \mathrm{~h}$, the cells were subjected to screen with Gentamycin analogue G418 (400 $\mu \mathrm{g} / \mathrm{ml}$ ) for $14 \mathrm{~d}$. G418-resistant clones were picked out for transient transfection. A $6.4 \mathrm{ml}$ of transfection solution containing $10 \mu \mathrm{g}$ of STAT3S727A mutant and $50 \mu \mathrm{l}$ of DOTAP in serum-free medium was added to each plate according to the manufacturer's instructions. Transfection was allowed to proceed at $37^{\circ} \mathrm{C}$ for $10 \mathrm{~h}$, followed by incubation in DMEM containing 20\% FCS without removing the transfection solution for an additional $20 \mathrm{~h}$.

\section{Northern blot [13]}

Total RNAs from the HSS-transfected, the pCDNA 3.1-transfected and the wild-type cells were extracted respectively. A total of $20 \mu \mathrm{g}$ RNAs from each group were denatured, electrophorized on $1.0 \%$ agarose gel containing $2.2 \mathrm{M}$ formaldehyde $/ 1 \times$ MOPS and transferred onto nitrocellulose membrane. The membrane was hybridized with radioactively labeled 373-bp probe of EcoR I/Sal I fragment of HSS cDNA. The radioactive signals were visualized by autoradiography on the Kodak X-Omat films (Eastman Kodak Co.) at $-70^{\circ} \mathrm{C}$. After exposure, the membrane was re-hybridized with ${ }^{32} \mathrm{P}$ probe of glyceraldehydes-3-phosphate dehydrogenase (GAPDH) to verify the uniformity of RNA loading.

\section{Cell growth assay}

The assay of cell growth was performed by non-radioactive photometry based upon the cellular conversion of the tetrazolium salt, MTS [3-(3,5-dimethylthialzol-2-yl)-5-(3 carboxymethoxypheny)2-(4-sulphophenyl)-2H-tetrazolium] into a formazan product which is soluble in culture medium[14]. Briefly, the HSS-transfected and vector-transfected HepG2 cells were cultured in serum-starved medium for $24 \mathrm{~h}$ for synchronization of cell cycle. Then the cells were trypsinized and inoculated into 96 -well plates $\left(5 \times 10^{3}\right.$ cells/well). Two hours prior to measurement, MTS reagent was added into the medium and the absorption values at $490 \mathrm{~nm}$ were determined with a Microplate Reader (Bio-Rad) .

\section{Western blot[18]}

Total cellular extracts were prepared as described previously[15]. In brief, cells were washed three times with cold PBS ( $\mathrm{pH} 7.4)$ and lyzed with lysis-buffer [150 mM NaCl, $50 \mathrm{mM}$ Tris- $\mathrm{HCl}$ ( $\mathrm{pH} 7.2$ ), $1 \%$ deoxycholic acid, $1 \%$ Triton X-100, 0.25 mM EDTA (pH 8.0), 5 $\mu \mathrm{g} / \mathrm{ml}$ leupeptin, $5 \mu \mathrm{g} / \mathrm{ml}$ aprotinin, $1 \mu \mathrm{g} / \mathrm{ml}$ pepstatin $\mathrm{A}, 1 \mathrm{mM}$ phenylmethylsulfony fluoride, $5 \mathrm{mM} \mathrm{NaV}$, and $100 \mu \mathrm{M}$ sodium orthovanadate]. Then the cell lysates were incubated on ice for $30 \mathrm{~min}$ and centrifuged at $10,000 \mathrm{~g}$ for $25 \mathrm{~min}$ at $4^{\circ} \mathrm{C}$, the supernatant containing the total cellular extracts was collected. Cytoplasmic extracts and nuclear extracts were prepared according to Bromberg et al [16]. For cytoplasmic extracts, cells were scraped and centrifuged at 11,500 rpm for $2 \mathrm{~min}$. The pellet was re-suspended in 3 volumes of cytoplasmic buffer [20 mM HEPES ( $\mathrm{pH} 7.9$ ), $10 \mathrm{mM} \mathrm{KCl}, 0.1 \mathrm{mM} \mathrm{NaV}$, $1 \mathrm{mM}$ EDTA, 10\% glycerol, $1 \mathrm{mM}$ DTT, $1 \mathrm{mM}$ PMSF] and left on ice for $10 \mathrm{~min}$, lysed using a Dounce homogenizer and checked microscopically for cell lysis. The lysates were centrifuged agian at $11,500 \mathrm{rpm}$ for $5 \mathrm{~min}$ and the supernatant containing cytoplasmic extracts was collected.The pellet was washed once with 3 volumes of cytoplasmic buffer, and re-suspended in 2 volumes of nuclear buffer [420 mM NaCl, 20\% glycerol, 20 mM HEPES (pH 7.9), 10 mM KCl, 1 mM EDTA, 0.1 mM NaV, 1 mM PMSF, 1 mM DTT], incubated on ice for $20 \mathrm{~min}$ and centrifuged again at 12,500 rpm for $10 \mathrm{~min}$. The supernatant containing nuclear protein was saved. Protein concentrations were determined by BCA assay[17].

$50 \mu \mathrm{g}$ of proteins from total, cytoplasmic or nuclear extracts were separated on $7 \%$ or $12 \%$ sodium dodecyl sulfate-polyacrylamide gel electrophoresis (SDS-PAGE) and electrotransferred onto nitrocellulos membrane. The membrane was blocked in 5\% non-fat milk, incubated with indicated antibodies (all at 1:1000 dilution) and washed with Tris-buffered saline containing $0.1 \%$ Tween 20 (TBST), followed 


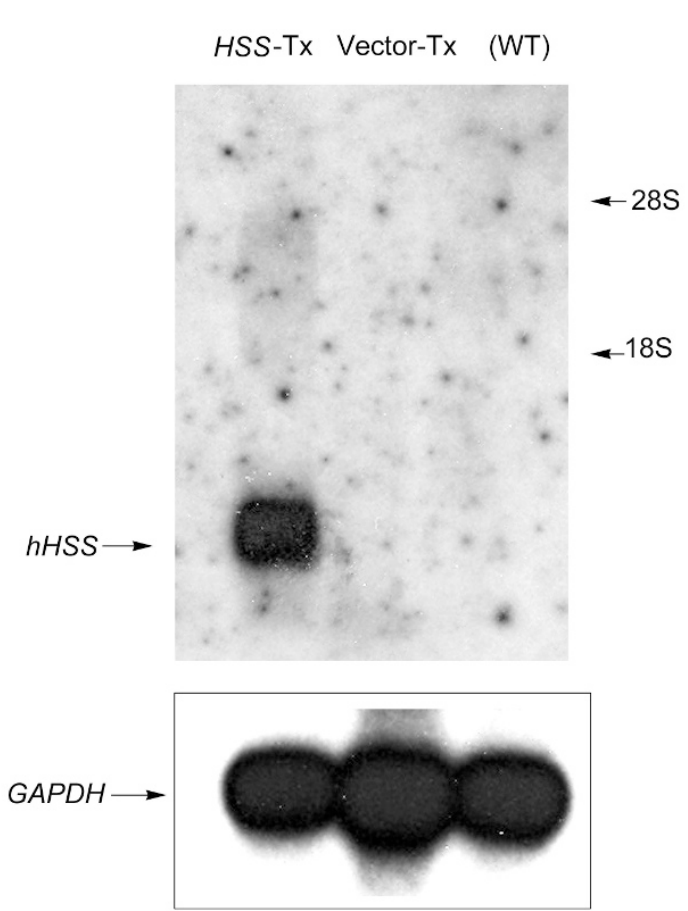

Fig 1. Expression of HSS. mRNA of the HSS-transfected (HSSTx), the vector-transfected (Vector-Tx) and wild-type HepG2 cells were extracted and hybridized with radioactive labeled $H S S$ probe. The blot was qualified using GAPDH as an internal control.

by incubation with peroxidase-conjugated goat anti-rabbit IgG for $1 \mathrm{~h}$. Then the membrane was washed again with TBST, detected by the enhanced chemiluminescence (ECL) and subjected to exposure on X-ray films. Densitometric analysis was performed using an image scanner (GS-700, Bio-Rad). For re-probing, the membrane was incubated in stripping buffer [2\% SDS, $62.5 \mathrm{mM}$ Tris- $\mathrm{HCl}(\mathrm{pH} 6.8)$, $100 \mathrm{mM} \beta$-mercaptoethanol] for $30 \mathrm{~min}$ at $50^{\circ} \mathrm{C}$, rinsed with TBST and redetected for total p44/42 MAP kinase or STAT3.

\section{Statistics}

All values were expressed as means $\pm \mathrm{SD}$. Statistical significance was determined by one-way ANOVA. P values $<0.05$ were considered significant.

\section{RESULTS}

\section{$H S S$ expression in $H S S$-transfected cells}

The result of Northern blot showed that HSS mRNA was expressed exclusively in HSS-transfected cells and was rarely detected either in the vector-transfected (pCDNA vector only) or in the wild-type HepG2 cells (Fig 1).

\section{Cell growth and ERK1/2 activation}

After transfection with HSS gene, the growth of HepG2 cells was found visibly accelerated as compared to that of the vector-transfected cells (Fig 2A). Consistantly, activa-

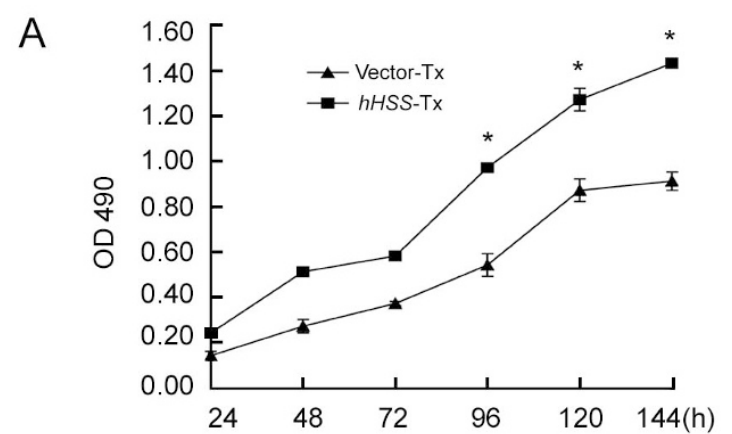

B
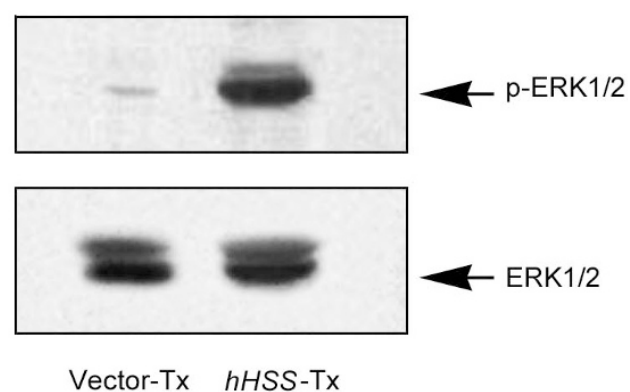

Fig 2. The inhibitory effect of activated-ERK $1 / 2$ on $h H S S$-transfected HepG2 cells. (A) The growth curves of the vector-transfected HepG2 cells (vector-Tx) and $h H S S$-transfected HepG2 cells ( $h H S S$ Tx) were assayed with MTT (see Materials and Methods). *represents $\mathrm{P}<0.05$ as compared to the vector transfected cells. (B) The threonine/tyrosine phosphorylation of ERK1/2 analyzed by Western blot in total cellular extracts of the cells. (Upper panel): The threonine/tyrosine phosphorylation of ERK1/2 (p-ERK1/2) detected by blotting with phospho-p44/42 MAP kinase antibody. (Lower pane10: Reprobing of the blots with p44/42 MAP kinase antibody which is against total 44/42 MAP kinase protein.

tion of ERK $1 / 2$ was enhanced in the HSS-transfected cells, but not in the vector-transfected cells (Fig 2B), indicating that the growth-promoting effect of HSS gene was closely related to ERK $1 / 2$ activation. The stimulatory effect of HSS on hepatoma cell line was also confirmed in another hepatoma cell line BEL-7402[11]. The consistence of these results dealt with HSS function suggested that this novel growth-stimulating substance exerted proliferative effect on hepatoma cells. Since ERK1/2 is the converging molecule which undergoes phosphorylation upon activations of a variety of signal molecules[19], it is reasonable to expect that the phoshorylated ERK1/2 might promote the transcriptions of growth-related genes after its translocating into the nucleus as an independent transcription factor or influence other probable latent transcription factors during the process of hepatocyte re-growth. If so, the regulatory effect of ERK $1 / 2$ on STAT3 activation as well as the translocation into the nucleus became our priority interest in the following investigations. 


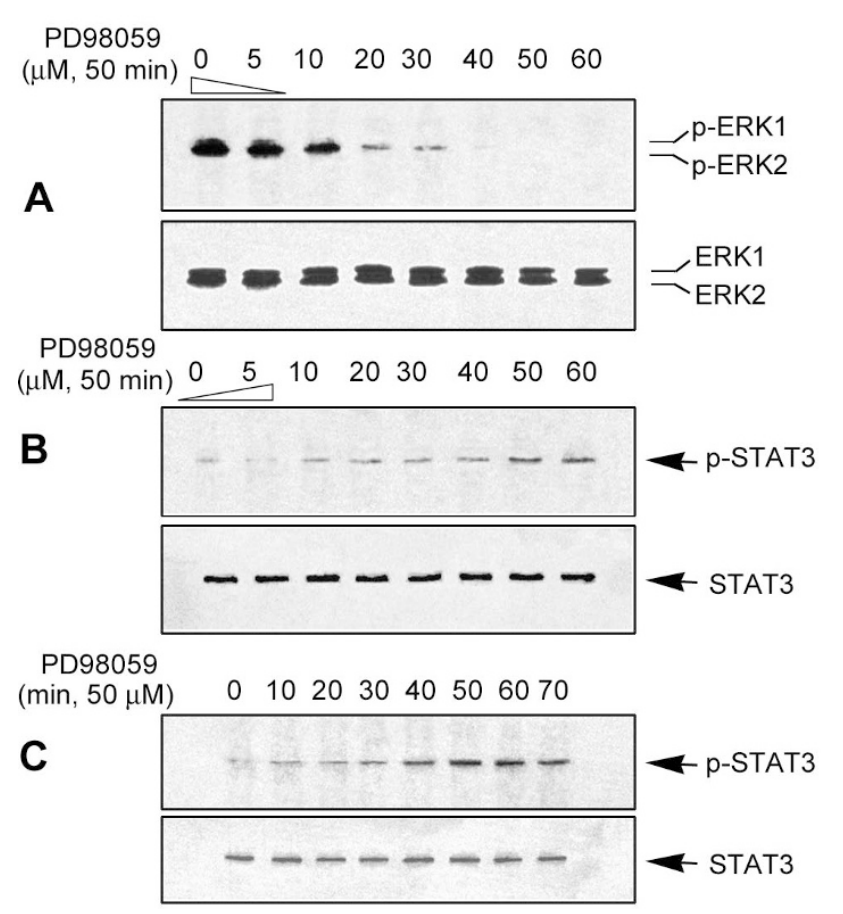

Fig 3. Inverse effect of PD 98059 on phosphorylation of ERK1/2 and STAT3. Total cellular extracts were prepared and analyzed by Western blot in the hHSS-transfected HepG2 cells. (A) Threonine/ tyrosine phosphorylation of ERK1/2 as detected by blotting with phospho-p44/42 MAP kinase antibody (upper panel) and re-probed with anti-p44/42 MAP kinase antibody (lower panel). (B) Tyrosine phosphorylation of STAT3 detected by blotting with phosphoSTAT3 antibody using the same lysates as A (upper panel) and reprobed with anti-STAT3 antibody (lower panel). (C) Time course of tyrosine phosphorylation of STAT3 after PD98059 treatment.

\section{Effect of PD98059 on ERK1/2 and STAT3 phos- phorylation}

As shown in Fig 3A, phosphorylations of ERK1/2 in the HSS-transfected cells were gradually reduced after treatment with increasing dose of PD98059. The maximum inhibition of PD98059 on ERK1/2 was found at $50 \mu \mathrm{M}$ when the phosphorylation completely disappeared. With regard to STAT3, its tyrosine phosphorylation did not show any visible up-regulation in the $H S S$-transfected cells without PD98059 administration (lane 1 in Fig 3B and 3C). To substantiate the influence of ERK1/2 on STAT3, the HSStransfected cells were treated either with increasing dosage of PD98059 $(0,5,10,20,30,40,50$ and $60 \mu \mathrm{M})$ or for extended exposure time $(50 \mu \mathrm{M}$ for $0,10,20,30,40$, $50,60,70 \mathrm{~min})$. The phosphorylations of both ERK1/2 and STAT3 were measured in parallel. Interestingly, it was found that STAT3 protein became tyrosine 705 phosphorylated, which was proportional to both dosages and exposure times to PD98059 (started from lane 3 in Fig 3B and C). Such augmented phosphorylation of STAT3

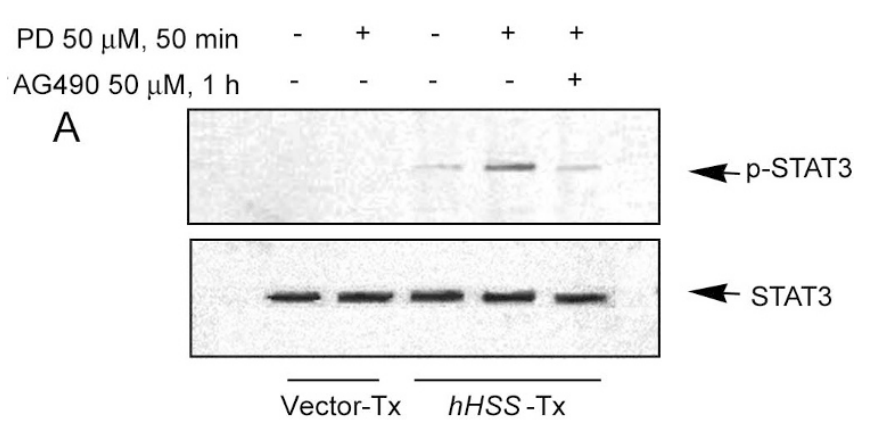

B

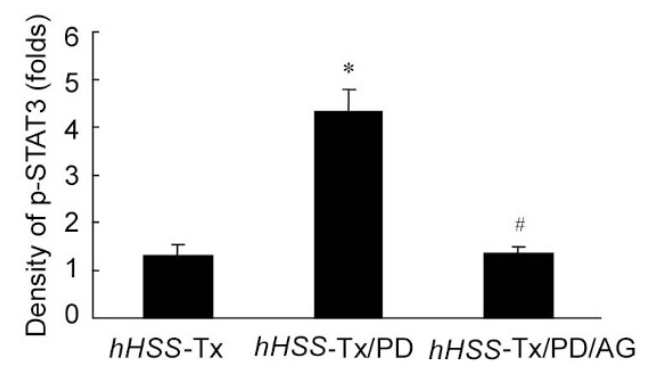

Fig 4. Inhibitory ability of AG490 on tyrosine phosphorylation of STAT3. Total cellular extracts were prepared and analyzed by Western blot in the vector-transfected HepG2 cells (vector-Tx) and the hHSS-transfected HepG2 cells (hHSS-Tx). (A) Tyrosine phosphorylation of STAT3 detected by blotting with phospho-STAT3 antibody (upper panel) and re-blotting with anti-STAT3 antibody which is against total STAT3 protein (lower panel). (B) Densitometric measurements of band intensity of tyrosine phosphorylated STAT3. Values are expressed as means \pm SD from four independent experiments. $\mathrm{PD}=\mathrm{PD} 98059 .{ }^{*} \mathrm{P}<0.01$ vs $h H S S-\mathrm{Tx},{ }^{*} \mathrm{P}>0.05$ vs $h H S S-\mathrm{Tx}$.

under ERK1/2 activation strongly suggests that some intrinsic correlations between ERK1/2 inhibition and STAT3 activation may exist.

AG490 was first described as a suppressor of leukemia cell growth via its inhibitory effect on JAKs[20] and has been recently defined as specific inhibitor of JAKs, upstream activator of STATs. Pretreatment of AG490 (50 $\mu \mathrm{M}, 1 \mathrm{~h}$ ) did not interfere with the phosphorylation of ERK1/2 (data not shown). However, exposure of PD98059-treated cells to AG490 $(50 \mu \mathrm{M}, 1 \mathrm{~h})$ resulted in notable decline in STAT3 phosphorylation (Fig 4). The inhibitory effect of AG490 on STAT3 phosphorylation in presence of PD98059 suggested that STAT3 was endogenously upregulated following ERK1/2 inactivation caused by PD98059.

\section{Nuclear translocation of STAT3 in HSS-transfected cells}

In response to cell stimulation, the latent cytoplasmic STATs are phosphorylated at tyrosine residues and subsequently translocated into nucleus. The STAT3 nuclear transloca- 


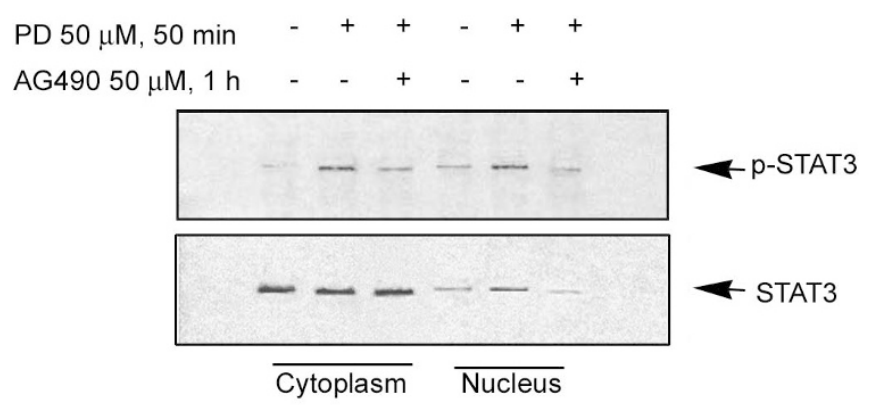

Fig 5. Requirement of PD98059 for the maximal nuclear translocation of STAT3. Cytoplasmic extracts (lanes 1-3) and nuclear extracts (lanes 4-6) were prepared and analyzed by Western blot in hHSS-transfected HepG2 cells. (Upper panel): Tyrosine phosphorylation of STAT3 detected by blotting with phospho-STAT3 antibody. (Lower panel): Re-blotting with anti-STAT3 antibody.

tions under ERK1/2 inactivation were observed by Western blotting of the phosphorylated STAT3 either in cytoplasmic or nuclear compartments. Consistent with Fig 4, inhibition of ERK1/2 caused by PD98050 (50 $\mu \mathrm{M}, 50 \mathrm{~min})$ could augment the STAT3 phosphorylation in both compartments (lane 2 and 5 in Fig 5). It should be noted that total STAT3 protein also increased in the nucleus (lane $4 v s$ 5 in lower panel of Fig 5). This result might be explained as that the STAT3 had been phosphorylated and thereafter accumulated into the nucleus. To some extent, the accumulation of phosphorylated STAT3 in the nucleus was attenuated by AG490 (50 $\mu \mathrm{M}, 1 \mathrm{~h})$, suggesting that the inhibition of ERK1/2 by PD98059 could promote the nuclear translocation of STAT3 in the HSS-transfected cells.

\section{Contribution of S727 phosphorylation to negative regulation of STAT3 by ERK1/2}

There are several hot sites within STAT3 molecule which potentially govern STAT3 tyrosine phosphorylation[21]. Among these hot sites serine at residual 727 (S727) has been widely studied. In order to elucidate whether S727 serves as key link between ERK1/2 and STAT3, we introduced a STAT3S727A mutant into the HSS-transfected cells and observed dominant negative STAT3 phosphorylation influenced by ERK1/2. Similar to Fig 4 and 5, an increase of tyrosine phosphorylation in the untransfected cells could be seen after inhibition of ERK1/2 (lane 1 vs 2 in Fig 6). More importantly, the mutant STAT3 phosphorylation was enhanced to the level that was comparable to that of PD98059 (lane 3 vs 2 in Fig 6). It had to be noted that the tyrosine phosphorylation in mutant STAT3 was obviously suppressed by JAK inhibitor AG490, but the suppression was not influenced by PD98059 (lanes 5-6 vs 1, Fig 6). The finding that STAT3 protein lacking S727 manifested tyrosine-phosphorylation in the HSS-transfected HepG2

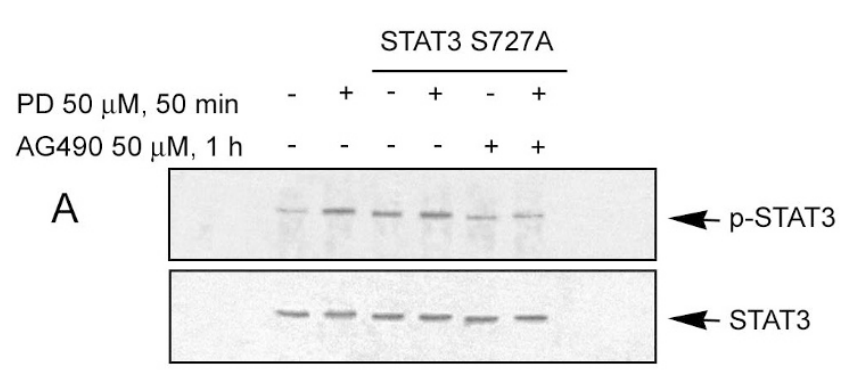

B

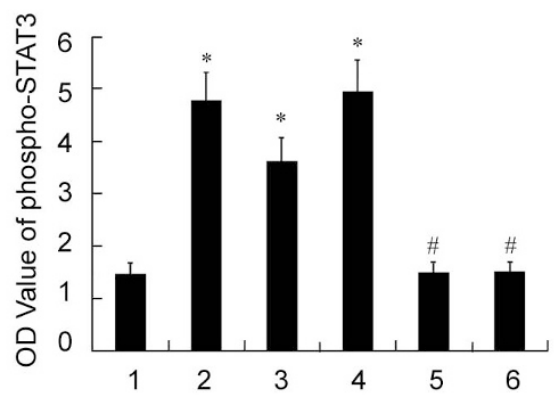

Fig 6. The role of STAT3 S727 phosphorylation in the regulation of STAT3 by ERK1/2. Total cellular extracts were prepared and analyzed by Western blot in hHSS-transfected HepG2 cells. (A) Tyrosine phosphorylation of STAT3 detected by blotting with phosphoSTAT3 antibody (upper panel) and re-blotting with anti-STAT3 antibody (lower panel). (B) Quantification of the phosphorylated STAT3. Values are expressed as means \pm SD from five independent experiments. The columns 1-6 in B are corresponding to lanes 1-6 in A. STAT3 S727A, a mutant of STAT3 in which S727 was substituted by Alanine. ${ }^{*} \mathrm{P}<0.05$ vs lane $1 .{ }^{*} \mathrm{P}>0.05$ vs lane 1 .

implied that STAT3 could be involved in the signaling of HSS, providing the possibility that either ERK1/2 activity was deprived or STAT3 was subjected to modificate itself.

\section{DISCUSSION}

The major finding of the present study is that the inhibition of ERK1/2 by PD98059 can augment STAT3 activation partially through STAT3 S727 phosphorylation in the HSS-transfected HepG2 cells. The regulation of ERK1/2 on STAT3 activitation might be due to phosphorylation of serine residual at 727 .

The STAT proteins are a family of latent transcription factors[22]. Upon receptor engagement, STATs become tyrosine phosphorylated, translocate to the nucleus and promote the expression of target genes. STATs are involved in two sets of signaling pathways, receptor tyrosine kinase (RTK) and JAK-STATs which are mainly activated by growth factors and cytokines. The cross-talk between these two signaling pathways is often encountered, and as a consequence there may exist some unpredictable, even contradictory effects within the same type of target cells. Among STAT family members, STAT3 has been widely studied in the regulation of liver regeneration[23]. Tyrosine 
phosphorylation of STAT3 is necessarily required for its dimer formation, nuclear translocation, and DNA binding. In addition to tyrosine phosphorylation, the maximal activation of this protein requires serine phosphorylation within the transactivation domain as well[24]. Therefore, elucidation of regulatory mechanism of STAT3 tyrosine phosphorylation will allow us to better understand the signaling pathway of HSS, a novel hepatic mitogen, as well as its physiological function.

There are contradictory conclusions on the modulation of MEK and ERK1/2 on STAT3. It was found in COS-1 cells that epidermal growth factor (EGF) and platelet-derived growth factor (PDGF) could stimulate MEK1, upstream activator of ERK1/2, leading to STAT3 tyrosine705 and serine-727 phosphorylation. Since it has already been convinced that interleukin-6 (IL-6) stimulated STAT3 phosphorylation via ERK1/2, numerous studies concerning with the interaction between ERK1/2 and STAT3 were frequently originated from the investigation of IL-6 signal transduction. The binding of IL- 6 to its cellular receptor activated simultaneously both ERK1/2 and STAT3[25, 26]. Ihara et al reported that stimulation of IL- 6 on the growth of PC12 cell via gp130 was exclusively dependent on ERK1/2 activation[27]. Furthermore, introducing mutant gp130 into cells resulted in an inactivation of ERK1/2, while the binding capacity of phosphorylated STAT3 to its specific DNA sequences was enhanced and the transcription of these targeted genes was remarkably increased[28]. Although sufficient evidences of positive regulations of ERK1/2 on STAT3 in IL-6 transduction have been reported, it was found that ERK1/2, on the other side, might exert a negative modulation on STAT3. Cao et al reported that MEK1-activated ERK2 could suppress the transcriptional activity of STAT3, which was represented by the inhibition of its DNA-binding activity and its tyrosine phosphorylation[29]. A similar study confirmed that constitutive expression of MEK1 in CHO cells inhibited the activation of STAT3 and hampered the binding of phosphorylated STAT3 to DNA[30]. As one explanation, Chung et al pointed out that paradoxical results concerning with MEK regulation on STAT3 were determined by whether ERK1/ 2 was involved in[31]. The dual regulation of ERK1/2 on STAT3 and interactive consequences varied according to different cell types and extracellular stimulators, and so on. In the present study, we found that HSS-induced ERK1/2 activation in HepG2 cells exerted negative modulation on STAT3 and elimination of ERK1/2 activation could restore STAT3 activity. Restoration of STAT3 tyrosine phosphorylation was further overwhelmed by JAK inhibitor (AG490), suggesting that the modulation of ERK1/2 on STAT3 seemed to be closely related to JAK.

In the present study we reported that HSS expression might stimulate STAT3 tyrosine phosphorylation if S727 in STAT3 was mutated. As a RTK-initiated signal, it had been postulated that STAT3 could be activated either directly by the receptor or indirectly through JAK proteins. However, the molecular basis for the negative regulation of STAT3 by ERK1/2 remains unsolved. There was a conserved carboxyl-terminal motif, PXSP (corresponding to S727 in STAT3) in STAT3 protein and ERK1/2 was capable to directly modify this motif. ERK2 phosphorylated STAT3 on three serine-containing peptides and decreased its tyrosine phosphorylation induced by EGF treatment[29]. In this case, serine phosphorylation was considered as a prerequisite for STAT3 maximal activation[32]. An increase in EGF-induced tyrosine phosphorylation of the STAT3S727A mutant compared to wild-type STAT3 has also been observed[27]. Since we previously demonstrated that HSS enhanced the phosphorylation of EGF receptor and stimulated Ras-MAPK cascade[8-11], here we reported that transfection of HSS significantly augmented the tyrosine phosphorylation of STAT3 if serine 727 was modified. It is predictable that HSS action on hepatocytes will accord itself with the defined feature of a growth factor. It has been known that STAT3 as downstream signal of IL-6 played an important role in liver regeneration, and deficiency in STAT3 signaling as observed in IL-6 knockout mice[33] would result in impaired lethality for liver regrowth[34]. If so, identification of HSS specific binding receptor on the hepatic cell membrane will draw a definitive resolution for complete outlook of this hepatic mitogen.

\section{ACKNOWLEDGMENT}

We thank Dr. Toshio Hirano (Department of Molecular Oncology (C7) Graduate School of Medicine, Osaka University, Japan) for the generally gift of STAT3S727A plasmid. We are grateful to Li CHEN for her technical assistance for Western blot. This work was supported by the National Natural Science Foundation of China (No. 39870285, No. 0070342).

Received, June 16, 2003

Revised, Dec 12, 2003

Accepted, Jan 8, 2004

\section{REFERENCES}

1 Michalopoulos GK, DeFrances MC. Liver regeneration. Science 1997; 276:60-6.

2 Fausto N. Liver regeneration. J Hepatol 2000; 32:19-31.

3 Ihle JN. STATs: signal transducers and activators of transcription. Cell 1996; 84(3):331-4.

4 Preface JR. STAT signaling. Oncogene 2000; 19:466-7.

5 Fausto N, Laird AD, Webber EM. Liver regeneration. 2. Role of growth factors and cytokines in hepatic regeneration. FASEB J 1995; 9(15):1527-36. 
6 Tomiya T, Ogata I, Yamaoka M, et al. The mitogenic activity of hepatocyte growth factor on rat hepatocytes is dependent upon endogenous transforming growth factor-alpha. Am J Pathol 2000; 157(5):1693-701.

7 Tomiya T, Ogata I, Fujiwara K. Transforming growth factor alpha levels in liver and blood correlate better than hepatocyte growth factor with hepatocyte proliferation during liver regeneration. Am J Pathol 1998; 153(3):955-61.

8 An W, Liu XJ, Lei TG, Du GG. Growth induction of hepatic stimulator substance in hepatocytes through its regulation on EGF receptors. Cell Res 1999; 9:37-49.

9 Dai J, An W, Gao DC, Chen L. Influence of hepatic stimulator substance on p21ras expression in human hepatic carcinoma cell BEL-7402. Acta Physiologica Sinica 2000; 52(3):225-9.

10 Chen L, An W, Tan X, Gao DC, Dai J. Phosphorylation of hepatic stimulator substance on mitogen-activated protein kinase in BEL-7402 hepatoma cells. Chin J Hepatology 2001; 9 (1):22-4.

11 Chen L, Sun H, An W. Production of recombinant hepatic stimulator substance and characterization of its growth promoting activity in hepatoma cells. Hepatology 2002; 36(4):443A.

12 Cepko C. Introduction of DNA into mammalian cells. In: Ausubel FM, Brent R, Kingston RE, eds. Short Protocols in Molecular Biology, $3^{\text {rd }}$ ed. New York: John Wiley \& Sons 1995, 1-49.

13 Alweine JC, Kemp DJ, Stark GR. Method for detection of specific RNAs in agarose gels by transfer to diabenzyloxymethylpaper and hybridization with DNA probes. Proc Natl Acad Sci USA 1977; 74:5350-6354.

14 Goodwin CJ, Holt SJ, Downes S, Marshall NJ. Microculture tetrazolium assays: a comparison between two new tetrazolium salts, XTT and MTS. J Immunol Methods 1995; 179(1):95103.

15 Jian N, Tong Z, Kee WH, Li W, Cao X. Protein kinase C dassociates with and phosphorylates STAT3 in an interleukin-6dependent manner. J Biol Chem 1999; 274:24392-400.

16 Bromberg JF, Wrzeszczynska MH, Devgan G, et al. STAT3 as an oncogene. Cell 1999; 98:295-303.

17 Smith PK, Krohn RI, Hermanson GT, et al. Measurement of protein using bicinchoninic acid. Anal Biochem 1985; 150:76-85.

18 Renart J, Sandoval IV. Western blot. Methods Enzymol 1984; 104:455.

19 Pelech SL, Sangsera JS. MAP kinase, charting the regulation pathway. Science 1992; 57:1355-6.

$20 \mathrm{Ni}$ Z, Lou W, Leman ES, Gao AC. Inhibition of constitutively activated Stat 3 signaling pathway suppresses growth of prostate cancer cells. Cancer Res 2000; 60:1225-8.

21 Decker T, Kovarik P. Serine phosphorylation of STATs. Oncogene 2000; 19:2628-37.

22 Leaman DW, Leung S, Li X, Stark GR. Regulation of STATdependent pathways by growth factors and cytokines. FASEB J 1996; 10(14):1578-88.

23 Li W, Liang X, Kellendonk C, Poli V, Taub R. STAT3 contributes to the mitogenic response of hepatocytes during liver regeneration. J Biol Chem 2002; 277(32):28411-7.

24 Wen Z, Zhong Z, Darnell JE Jr. Maximal activation of transcription by Stat 1 and Stat 3 requires both tyrosine and serine phosphorylation. Cell 1995; 82(2):241-50.

25 Taga T, Kishimoto T. Gp130 and the interleukin- 6 family of cytokines. Annu Rev Immunol 1997; 15:797-819.

26 Kishimoto T, Taga T, Akira S. Cytokine signal transduction. Cell 1994; 76:253-62.

27 Ihara S, Nakajima K, Fukada T, et al. Dual control of neurite outgrowth by STAT3 and MAP kinase in PC12 cells stimulated with interleukin-6. EMBO J 1997; 16:5345-52.

28 Kim H, Hawley TS, Hawley RG, Baumann H. Protein tyrosine phosphatase 2 (SHP-2) moderates signaling by gp130 but is not required for the induction of acute-phase plasma protein genes in hepatic cells. Mol Cell Biol 1998; 18:1525-33.

29 Jain N, Zhang T, Fong SL, Lim CP, Cao X. Repression of Stat3 activity by activation of mitogen-activated protein kinase (MAPK). Oncogene 1998; 17(24):3157-67.

30 Sengupta TK, Talbot ES, Scherle PA, Ivashkiv LB. Rapid inhibition of interleukin- 6 signaling and STAT3 activation mediated by mitogen-activated protein kinases. Proc Natl Acad Sci USA 1998; 95:11107-12.

31 Cheung J, Uchida E, Grammer TC, Blenis J. STAT3 serine phosphorylation by ERK-dependent and -independent pathways negatively modulates its tyrosine phosphorylation. Mol Cell Biol 1997; (11):6508-16.

$32 \mathrm{Ng} \mathrm{J}$, Cantrell D. STAT3 is a serine kinase target in T lymphocytes. Interleukin 2 and $\mathrm{T}$ cell antigen receptor signals converge upon serine 727. J Biol Chem 1997; 272:24542-9.

33 Cressman DE, Greenbaum LE, DeAngelis RA, et al. Liver failure and defective hepatocyte regeneration in interleukin-6-deficient mice. Science 1996; 274:1379-83.

34 Hirano T, Ishihara K, Hibi M. Role of STAT3 in mediating the cell growth, differentiation and survival signals relayed through IL-6 family of cytokine receptor. Oncogene 2000; 19:2548-56. 